\title{
Multidisciplinary management of type 2 diabetes in children and adolescents
}

\author{
This article was published in the following Dove Press journal: \\ Journal of Multidisciplinary Healthcare \\ 27 July 2010 \\ Number of times this article has been viewed
}

\author{
Michael E Bowen ${ }^{1,2}$ \\ Russell L Rothman ${ }^{2,3}$ \\ 'Veterans Affairs Quality Scholars \\ Fellowship Program, Tennessee \\ Valley Healthcare System, Tennessee \\ Valley Geriatric Research Education \\ Clinical Center, Nashville, TN, USA; \\ ${ }^{2}$ Division of General Internal Medicine \\ and Public Health, Department of \\ Medicine, ${ }^{3}$ Vanderbilt Eskind Diabetes \\ Center, Vanderbilt University School \\ of Medicine, Nashville, TN, USA
}

\begin{abstract}
Although once considered a disease of adults, the prevalence of type 2 diabetes in youth is increasing at a significant rate. Similar to adults, youth with type 2 diabetes are at increased risk for developing hypertension, lipid abnormalities, renal disease, and other diabetes-related complications. However, children and adolescents with type 2 diabetes also face many unique management challenges that are different from adults with type 2 diabetes or children with type 1 diabetes. To deliver safe, effective, high-quality, cost-effective health care to adolescents with type 2 diabetes, reorganization and redesign of health care systems are needed. Multidisciplinary health care teams, which allow individuals with specialized training to maximally utilize their skills within an organized diabetes treatment team, may increase efficiency and effectiveness and may improve outcomes in children with type 2 diabetes. This review article provides a brief review of type 2 diabetes in children and adolescents, provides an overview of multidisciplinary health care teams, and discusses the role of multidisciplinary health care management in youth with type 2 diabetes.
\end{abstract}

Keywords: adolescent, type 2 diabetes, multidisciplinary

\section{Introduction}

The prevalence of type 2 diabetes in children and adolescents is increasing worldwide. ${ }^{1-3}$ The potential cumulative morbidity and mortality resulting from early-onset type 2 diabetes is staggering. However, to date, little evidence on the pathophysiology, management, complications, and long-term outcomes of type 2 diabetes in youth is available. As a result, experience from adults with type 2 diabetes or children with type 1 diabetes has been extrapolated to adolescents, but emerging evidence suggests that there are important differences in the disease between these populations. ${ }^{4}$ In order to curb the rising epidemic, novel disease management strategies are needed with a focus on care organization, delivery, and patient and family behavioral modification. These challenges are best approached by an organized, multidisciplinary health care team focused on delivering high-quality patient care. This review seeks to provide an overview of type 2 diabetes in youth, describe important components of multidisciplinary health care teams, and provide recommendations for future research.

\section{Classification of diabetes in youth}

Diabetes is classically conceptualized into 2 distinct entities: type 1 diabetes and type 2 diabetes. Type 1 diabetes results from an absolute deficiency in insulin secretion due to cell-mediated autoimmune destruction of pancreatic $\beta$-cells. Type 2 diabetes results from a combination of insulin resistance and inadequate insulin secretion. ${ }^{5}$ Classically, 
patients with type 1 diabetes present with thin body habitus, lack of insulin production, presence of autoantibodies to insulin or pancreatic islet cells, and ketoacidosis, whereas patients with type 2 diabetes present with overweight, evidence of increased insulin or insulin resistance, lack of autoantibodies, and no ketoacidosis. However, differentiation between type 1 and type 2 diabetes can be more challenging in pediatric patients. ${ }^{4,6-8}$ Among pediatric patients clinically diagnosed with type 2 diabetes, up to $33 \%$ have ketoacidosis at the time of presentation. ${ }^{6}$ Although autoantibodies are classically present in type 1 diabetes, up to $4 \%-7 \%$ of children with newly diagnosed type 1 diabetes are autoantibody negative, ${ }^{9}$ and $10 \%-75 \%$ of children with clinically diagnosed type 2 diabetes have detectable autoantibodies. ${ }^{6,10,11}$ Additionally, up to one-fourth of patients with type 1 diabetes present overweight. ${ }^{1}$

\section{Epidemiology of type 2 diabetes in children and adolescents}

Epidemiologic studies in type 2 diabetes are limited by the challenges of correct clinical classification of diabetes. Numerous reports have demonstrated global, dramatic increases in pediatric patients with type 2 diabetes, although the rate of increase varies widely between countries and ethnic groups. Most studies are based on case series, clinic cohorts, or registry data. Thus, the incidence and prevalence estimates may be subject to bias. The Pima Indians in North America have the world's highest reported incidence of diabetes ${ }^{12}$ with an estimated prevalence of type 2 diabetes of 5,100 per 100,000 adolescents in the 1990 s. ${ }^{8,13}$ Subsequently, North American case series have found that type 2 diabetes accounts for $8 \%-46 \%$ of diabetes in newly diagnosed patients aged 0-19 years. ${ }^{7,8}$ Population-based studies have found more modest prevalence estimates. The National Health and Nutrition Examination Survey III (NHANES III) estimated a diabetes prevalence (unknown type of diabetes) of 4,100 per 100,000 adolescents aged 12-19 years in the United States. ${ }^{7}$ The SEARCH for Diabetes in Youth Study conducted population-based ascertainment of diabetes in youth younger than 20 years in six geographically diverse regions of the United States. In this study, the overall prevalence of type 2 diabetes was 22 per 100,000 youth, whereas overall incidence was 24.3 per 100,000 person-years. Incidence and prevalence of type 2 diabetes in youth were highest among ethnic minorities and observed to increase with age. ${ }^{14,15}$

International studies have also demonstrated an increasing incidence and prevalence of type 2 diabetes in youth, although the rate of increase appears to be less than that observed in North
American studies. ${ }^{12}$ In Japan, the incidence of type 2 diabetes observed in a school-based urine glucose screening program was 3 per 100,000 youth, with more than $80 \%$ of children with type 2 diabetes being obese. ${ }^{16}$ A nationwide diabetes screening program among school children in Taiwan found that $54 \%$ of newly diagnosed diabetics were type 2 , with an incidence rate of 6.5 per 100,000. ${ }^{17}$ Between 2001 and 2006, the incidence rate of type 2 diabetes in the Australasian Paediatric Endocrine Group New South Wales (NSW) Diabetes Register was 2.5 per 100,000 person-years, with type 2 diabetes accounting for $11 \%$ of incident diabetes diagnoses in youth aged $10-18$ years. ${ }^{18}$ European incidence estimates of type 2 diabetes range from $0.25-1.52$ per 100,000 youth. ${ }^{12,19}$

Although the incidence and prevalence of type 2 diabetes in youth are increasing, the rates of increase do not currently appear to be of "epidemic" proportions. ${ }^{20}$ The appearance of type 2 diabetes in adolescents seems to mirror the increase in type 2 diabetes in adult populations throughout the world, although the appearance and rate of increase in children lag behind what has been observed in adults. Attention to the epidemiology of type 2 diabetes in adults may predict the patterns of emergence of type 2 diabetes in adolescents. ${ }^{12}$

\section{Risk factors}

Risk factors for type 2 diabetes in children have been identified largely from case series, patient or disease registries, and clinic-based cohorts. The well-characterized risk factors include race or ethnicity, family history, obesity, physical inactivity, low birth weight, intrauterine exposure to maternal diabetes, puberty, gender, and conditions predisposing to insulin resistance such as polycystic ovarian syndrome. , $^{2,21-25}$

Irrespective of country of residence, children of American Indian, Alaskan Native, Asian American, Pacific Islander, Hispanic, and African American ethnic groups have the highest rates of type 2 diabetes, with American Indian and Canadian First Nation youth having the highest prevalence overall. ${ }^{7,26}$ Globally, Indo-Asians appear to be the most at risk group, and this may be related to a tendency toward central adiposity. ${ }^{21}$ African American children have higher insulin levels in response to an oral glucose tolerance test (OGTT) when compared with white children, even after adjustment for weight, age, and pubertal stage. ${ }^{8,27}$ Data suggest that minority children may have a genetic predisposition to insulin resistance that interacts with environmental modulators and predisposes to the development of type 2 diabetes. ${ }^{8}$

Between $45 \%$ and $80 \%$ of pediatric patients with type 2 diabetes have at least one parent with diabetes, and $74 \%-100 \%$ of children have a first- or second-degree 
relative with type 2 diabetes. ${ }^{2,25}$ Japanese children with type 2 diabetes demonstrate familial clustering, with $48 \%-60 \%$ of parents having diabetes and siblings having a 175- to 250 -fold increase in diabetes when compared with the general population. ${ }^{2}$ Physical, behavioral, and environmental risk factors appear to be pervasive throughout the family. Adolescents with type 2 diabetes often belong to high-risk family units, having parents and siblings with increased central obesity, increased rates of type 2 diabetes and insulin resistance, and diets high in fat and low in fiber. ${ }^{28}$

Obesity may be the most important determinant of insulin resistance and type 2 diabetes. ${ }^{25}$ Globally, the risk in type 2 diabetes mirrors urbanization and economic development, and obesity appears to be the key link. ${ }^{2}$ The incidence of type 2 diabetes in Japan paralleled the increasing prevalence of obesity in school children from 1974 to $1995 .^{2,29}$ This has also been observed in China, Hong Kong, England, Australia, and the United States. ${ }^{2}$ Up to $85 \%$ of children presenting with type 2 diabetes are obese or overweight, ${ }^{21}$ and adiposity accounts for approximately $50 \%$ of the variance seen in insulin sensitivity. ${ }^{25}$ The impact of overweight and obesity is further compounded by decreased physical activity, decreased participation in physical education, and increased television viewing commonly observed in this population. ${ }^{2}$ Dietary behaviors can contribute to excessive weight gain and may also contribute to the development of type 2 diabetes in children. ${ }^{2}$ In adults, consumption of sugar-sweetened beverages has been associated with weight gain, obesity, and risk for diabetes. ${ }^{30,31}$

Other potential risk factors for the development of type 2 diabetes include low or high birth weight, rapid weight gain, puberty, and gender. Low birth weight, high birth weight, and maternal diabetes are associated with increased risk of type 2 diabetes in Pima Indian youth. ${ }^{7,32}$ Low birth weight has been associated with increased risk for glucose intolerance, type 2 diabetes, and metabolic syndrome in adult life, although the mechanisms remain largely unknown. ${ }^{33}$ Additionally, rapid weight gain in early childhood is a risk factor for subsequent obesity and type 2 diabetes in adulthood. ${ }^{25}$ Puberty confers a state of relative insulin resistance and also increases basal and stimulated insulin responses. ${ }^{25}$ Although little gender variation is observed in adults with type 2 diabetes, adolescent girls are nearly twice as likely to develop type 2 diabetes compared with boys. ${ }^{2,3}$

\section{Diagnosis}

Diabetes diagnostic criteria are identical in children and adults (Table 1). The presence of a random plasma glucose level $\geq 200 \mathrm{mg} / \mathrm{dL}(\geq 11.1 \mathrm{mmol} / \mathrm{L})$ associated with classic
Table I Diagnostic tests for impaired glucose regulation and diagnosis of diabetes ${ }^{5,32}$

\begin{tabular}{|c|c|c|c|}
\hline & Test & & \\
\hline & $\begin{array}{l}\text { Fasting blood } \\
\text { glucose },^{\mathrm{a}} \mathrm{mg} / \mathrm{dL} \\
(\mathrm{mmol} / \mathrm{L})\end{array}$ & $\begin{array}{l}\text { 2-h plasma } \\
\text { glucose, }{ }^{\mathrm{b}} \mathrm{mg} / \mathrm{dL} \\
(\mathrm{mmol} / \mathrm{L})\end{array}$ & $\begin{array}{l}\text { Hemoglobin } \\
\text { Alc }\end{array}$ \\
\hline Normal & $<100(<5.6)$ & $<140(<7.8)$ & - \\
\hline $\begin{array}{l}\text { Impaired } \\
\text { fasting } \\
\text { glucose }\end{array}$ & $100-125(5.6-6.9)$ & - & - \\
\hline $\begin{array}{l}\text { Impaired } \\
\text { glucose } \\
\text { tolerance }\end{array}$ & - & |40-199 (7.8-|| I.0) & - \\
\hline Diabetes & $\geq 126(\geq 7.0)$ & $\geq 200(\geq \mid I . I)$ & $\geq 6.5$ \\
\hline
\end{tabular}

Notes: a Minimum 8-h fast; ${ }^{b}$ Following a 75-g oral glucose tolerance test (OGTT).

symptoms of hyperglycemia (polyuria, polydipsia, and unexplained weight loss), plasma glucose level $\geq 200 \mathrm{mg} / \mathrm{dL}$ ( $\geq 11.1 \mathrm{mmol} / \mathrm{L}$ ) 2 hours after a 75 -g OGTT, or fasting plasma glucose level $\geq 126 \mathrm{mg} / \mathrm{dL}$ ( $\geq 7.0 \mathrm{mmol} / \mathrm{L}$ ) on two separate days confirms a diagnosis of diabetes. ${ }^{5,34}$ Additionally, in 2009, hemoglobin $\mathrm{A} 1 \mathrm{c}\left(\mathrm{HbA}_{1 \mathrm{c}}\right)$ was added as a diagnostic tool. ${ }^{34}$ Individuals with $\mathrm{HbA}_{1 \mathrm{c}}$ between $6.0 \%$ and $6.5 \%$ can be considered "at risk," while $\geq 6.5 \%$ can be considered a new diagnosis of diabetes. ${ }^{34}$

Although widely accepted, these diagnostic criteria are based on data from adult populations and extrapolated to children and adolescents. Mounting evidence suggests that pathology and physiology of disease differ between children and adults. ${ }^{2}$ Fasting plasma glucose level and OGTT have not been evaluated in rigorous, large studies in children. The correct "dose" of glucose for the OGTT and the expected response in children are unknown. Additionally, the impact of variation in glucose metabolism throughout childhood, including increased insulin resistance during the pubertal period, is unknown and unaccounted by current diagnostic methods. ${ }^{21}$ Further research is needed to determine the performance of current diagnostic criteria for diabetes in children and adolescents.

\section{Screening}

Screening programs for type 2 diabetes seek to identify asymptomatic individuals who are likely to have disease. Onset of type 2 diabetes in childhood results in longer duration of disease, increased medical costs, higher lifetime rates, and earlier development of microvascular and macrovascular complications. ${ }^{2}$ Although routine screening for type 2 diabetes in children seems intuitive, the overall prevalence of type 2 diabetes and the prevalence of undiagnosed disease are low. ${ }^{4}$ General screening programs of school-age children 
in Japan and Taiwan cost nearly US $\$ 10,000$ per case of type 2 diabetes identified. ${ }^{2}$ Relatively low disease prevalence and the high costs of universal screening have led to targeted screening in high-risk groups.

Current recommendations supported by the American Diabetes Association (ADA) and the International Diabetes Federation support the targeted screening of high-risk children and adolescents, beginning at 10 years of age or the onset of puberty, who meet the criteria outlined in Table 2., ${ }^{1,235}$ Based on convenience, cost, and ease of performance, fasting plasma glucose test, rather than the OGTT, is the preferred screening test. ${ }^{2,35}$

Strict utilization of these screening recommendations would lead to screening of approximately $10 \%$ of youth or approximately 2.5 million adolescents between the ages of 12 and 19 years in the United States. An estimated 5\% of screened youth may have impaired fasting glucose or undiagnosed diabetes. ${ }^{2}$ Given the low prevalence of type 2 diabetes in children and adolescents, screening tests are more likely to identify children with prediabetes rather than those with diabetes. This is suggested by NHANES data from the United States where between 1999 and 2000, no cases of type 2 diabetes were identified among adolescents aged 12-19 years, but nearly $18 \%$ of adolescents with obesity had impaired fasting glucose. ${ }^{36}$ Currently, effective management and treatment strategies of prediabetic states in children are unknown, ${ }^{4}$ and there is a lack of evidence that screening for type 2 diabetes in childhood decreases disease-related morbidity and mortality or demonstrates cost-effectiveness. ${ }^{35}$ Currently, adherence to screening guidelines is sporadic, with $<50 \%$ of children meeting screening criteria receiving screening and utilization of a random plasma glucose rather than fasting plasma glucose or OGTT. ${ }^{37}$

Table 2 Screening recommendations for type 2 diabetes in highrisk children and adolescents $1,2,35$

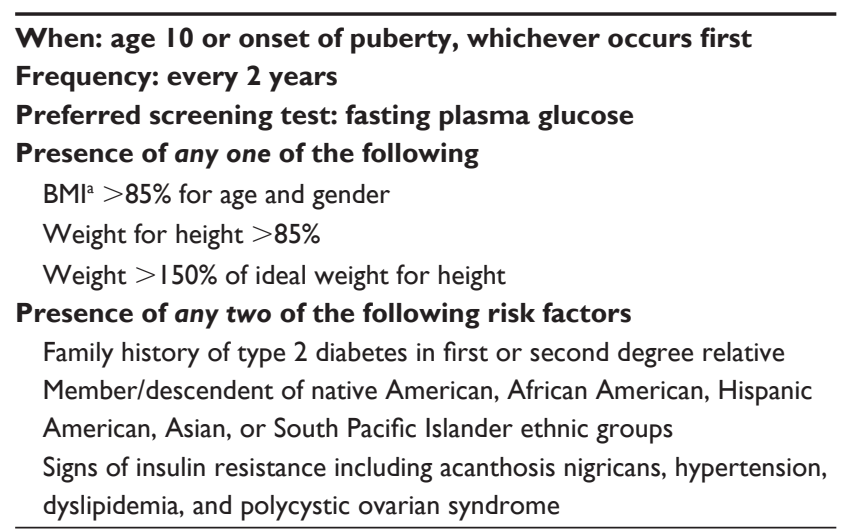

Abbreviation: BMI, body mass index.

\section{Complications}

Onset of type 2 diabetes in adolescence may place the individuals at risk for increased morbidity and mortality during their most productive life-years; however, limited longterm follow-up studies describing secondary complications of type 2 diabetes in children and adolescents are available.

Young adults aged 18-44 years with type 2 diabetes develop microalbuminuria and cardiovascular disease more rapidly than individuals diagnosed after age 45 and have a 14-fold relative increase in myocardial infarction risk compared with age-matched and gender-matched controls without diabetes..$^{38}$ In spite of shorter disease duration, many studies have demonstrated the greater prevalence of microalbuminuria in adolescents with type 2 diabetes compared with individuals with type 1 diabetes. ${ }^{39}$ Among Pima Indians with incident type 2 diabetes, microalbuminuria was present in $22 \%$ of adolescents at diagnosis and $58 \%$ after 10 years of follow-up. ${ }^{40}$

Although retinopathy remains more frequent in patients with type 1 diabetes, it may be present in adolescents with type 2 diabetes at the time of diagnosis, and patients with type 2 diabetes have a notably shorter duration of disease prior to its appearance. ${ }^{41} \mathrm{Up}$ to $9 \%$ of patients diagnosed with type 2 diabetes before age 30 have evidence of retinopathy at diagnosis, and nearly $13 \%$ develop proliferative retinopathy before age $35 .{ }^{42}$ Limited data suggest that rates of peripheral and autonomic neuropathy do not differ among adolescents with type 1 and type 2 diabetes; however, adolescents with type 2 diabetes appear to develop neuropathy at a more rapid rate. ${ }^{41,43}$

The presence of comorbid conditions, such as hypertension, dyslipidemia, and nonalcoholic fatty liver disease (NAFLD) is well documented in adolescents with type 2 diabetes. These illnesses are intimately associated with obesity and exist concurrently with diabetes in many patients. Hypertension is eight times more prevalent at the time of diagnosis in adolescents with type 2 diabetes compared with those with type 1 diabetes, with prevalence estimates ranging from $10 \%-32 \%{ }^{39}$ Dyslipidemia is also common with $18 \%-46 \%$ of adolescents with type 2 diabetes having elevated lowdensity lipoproteins and $29 \%-61 \%$ of individuals having elevated triglycerides. ${ }^{39}$ Elevated liver enzymes have been noted in up to $48 \%$ of adolescents with type 2 diabetes. In the setting of obesity, insulin resistance, dyslipidemia, and type 2 diabetes, these findings are often associated with NAFLD. ${ }^{39}$ Development of such complications may be related to poor clinical follow-up as up to $60 \%$ of adolescents with type 2 diabetes lack regular follow-up and have been noted to have 
higher body mass indexes and blood pressures and greater lipid abnormalities compared with adolescents receiving regular clinical care. ${ }^{44}$

\section{Management strategies: medications}

Effective treatment of type 2 diabetes requires a combination of lifestyle modification and medications. At present, data supporting the safe and effective treatment of type 2 diabetes in children and adolescents are sparse. As a result, treatments demonstrated to be effective in adults with type 2 diabetes have typically been extrapolated to adolescents. The ADA and International Diabetes Federation suggest initial trials of diet modification and exercise therapy in adolescents with type 2 diabetes. However, it is accepted that this is effective in $<10 \%$ of adolescents, and most patients will ultimately require pharmacological treatment. ${ }^{2,22}$

Metformin has been proven safe and effective in lowering fasting plasma glucose and $\mathrm{HbA}_{1 \mathrm{c}}$, and it is the recommended first line medication for treatment of type 2 diabetes in adolescents. Metformin remains the only US Food and Drug Administration (FDA)-approved agent for treatment of type 2 diabetes in the pediatric population. ${ }^{45}$ Although not approved by FDA for pediatric use in the United States, glimepiride lowers $\mathrm{HbA}_{1 \mathrm{c}}$ to an equivalent degree as metformin. However, glimepiride is associated with greater weight gain and more frequent hypoglycemia than metformin. ${ }^{46}$ To date, there are no long-term trials demonstrating safety and efficacy of insulin in pediatric patients with type 2 diabetes, although it is commonly utilized as initial therapy in symptomatic patients and in those presenting with diabetic ketoacidosis., Other medications for treatment of type 2 diabetes in adults, including sulfonylureas, thiazolidinediones, $\alpha$-glucosidase inhibitors, dipeptidyl peptidase IV inhibitors, incretin mimetics, and glucagon-like peptide-1 inhibitors, are rarely used in the treatment of adolescents with type 2 diabetes. Further studies evaluating the safety, efficacy, and long-term outcomes for the treatment of type 2 diabetes in adolescents are needed. The Treatment Options for Type 2 Diabetes in Youth (TODAY) study is a multicenter study enrolling 750 children and comparing metformin alone vs metformin plus rosiglitazone vs metformin plus intensive lifestyle intervention. This study is slated for completion in 2011. ${ }^{4,25}$

Although treatment with medications plays an important role in type 2 diabetes, the effectiveness of oral medications will inevitably diminish as disease progresses, and insulin will be required. It has been well demonstrated that adolescents with type 1 diabetes have inconsistent medication adherence in spite of potentially life-threatening consequences of nonadherence to treatment. Thus, the prospects of longterm medication adherence in adolescents with relatively asymptomatic type 2 diabetes are likely to be even lower. ${ }^{47}$ Nonpharmacologic interventions focusing on nutrition, activity, and lifestyle change may provide durable treatment options for adolescents with type 2 diabetes, provided they are sustained over a period of years. This may be particularly powerful in youth if modifications are adopted during the period in which healthy lifestyle habits are developed. At present, there is little evidence or research on the best approach to delivering and incorporating these aspects into patient care. Utilization of multidisciplinary care teams to provide comprehensive disease management may be useful.

\section{Principles of multidisciplinary care}

Provision of ongoing, comprehensive care for chronic medical illnesses such as type 2 diabetes presents unique challenges to our current medical system, which is generally designed and structured to respond to acute illness. Structural adaptations to provide care for chronic diseases such as type 2 diabetes rely on periodic follow-up visits to assess the effects of prior interventions and develop ongoing or modified plans of care. In current medical systems, patients may or may not receive diabetes education, self-management support, and services to enhance self-efficacy and activation, and if such services are received, they are often not interrelated or integrated into routine medical care.

Multidisciplinary approaches to the management of type 2 diabetes in children and adolescents build on the foundation of the "medical home". Initially described by the American Academy of Pediatrics (AAP) in $1992,{ }^{48}$ the patient-centered medical home $(\mathrm{PCMH})$ serves as a model for how practices should respond to all patients within a practice and has evolved substantially in the past decade with various degrees of adoption and implementation throughout pediatric and adult practices. At present, a unifying description and definition of the PCMH is lacking, although most incorporate elements of continuity of care, patient-centered care, enhanced access, improved communication between patients and providers, integrated care, coordinated care, and increased use of information technology. ${ }^{49,50}$ Although full implementation and evaluation have been limited, the PCMH may be particularly useful in management of chronic childhood diseases, and evidence suggests that the medical home improves health outcomes in children with chronic illness. ${ }^{48}$

The chronic care model $(\mathrm{CCM})^{51}$ provides a guide for multidisciplinary, team-based management of chronic 
disease and may be a useful model for adolescents with type 2 diabetes. The CCM is built upon the idea that effective chronic illness management requires comprehensive, systematic change rather than simply adding new features to an unchanged system focused on delivery of acute care. Chronic care delivered in a high-quality system necessitates continuous relationships with a care team, individualization of care according to patient needs and values, anticipation patient needs, cooperation among clinicians, and evidencebased care. ${ }^{51}$ The CCM also seeks to deliver patient-centered care, which considers an individual patient's personal preferences, values, lifestyle, family, and cultural traditions when formulating health care plans. By incorporating the patient and family members into clinical decision making, disease management is shared by patients and clinicians. As a result, a primary goal of the multidisciplinary health care team is to foster independent self-care responsibility that is reflected in self-monitoring, problem solving, and lifestyle choices in the patients. Thus, rather than dictating delivery of care, health care providers become guides, resources, and coaches to empower patients in disease management. ${ }^{52}$ Adoption of these principles into medical practice may help achieve the "triple aim" of health care: improving the individual experience of care, improving the health of populations, and reducing per capita cost of care for populations. ${ }^{53}$

Medical home demonstration projects sponsored by the Centers for Medicare and Medicaid Services (CMS) in the United States, private insurers, and private funding are ongoing and incorporate elements of both traditional PCMHs and the CCM. While the PCMH generally includes open access and care coordination among providers as key elements, the CCM does not account directly for these elements. Thus, the PCMH may be viewed as a framework for the implementation of the CCM. ${ }^{49}$ At this time, it is unclear whether the PCMH, the CCM, or an integrated approach achieves optimal outcomes in children with type 2 diabetes.

Additional considerations are important in adapting these recommendations into disease management strategies for children and adolescents with type 2 diabetes. Provision of high-quality pediatric care must account for the developmental stages of childhood and their relationship to diabetes understanding and diabetes management skills. The "4D model" describes unique considerations in the design, delivery, and evaluation of quality improvement and health services research in children. The 4 Ds include (1) Developmental change, (2) Dependency, (3) Differential epidemiology, and (4) Demographic patterns. ${ }^{54}$ Consideration of these factors will help facilitate the development of age-appropriate, multidisciplinary health care strategies for children with type 2 diabetes.

\section{Structure of the multidisciplinary health care team}

In adult patients, multifaceted disease management programs have been shown to improve patient satisfaction, patient adherence, and disease control across a variety of chronic medical conditions including diabetes. ${ }^{55,56}$ In type 2 diabetes, multidisciplinary disease management programs targeting both providers and adults have been shown to significantly decrease $\mathrm{HbA}_{1 \mathrm{c}}$ and increase patient self-care practices and self-efficacy. ${ }^{57,58}$ Intensive management within the primary care setting utilizing care coordinators and clinical pharmacists decreases $\mathrm{HbA}_{1 \mathrm{c}}$ and cardiovascular risk factors in adults with type 2 diabetes, ${ }^{59}$ with modest labor and program costs. ${ }^{60}$ Sustained reductions in $\mathrm{HbA}_{1 \mathrm{c}}$ have been shown to provide significant reductions in health care costs and utilization. ${ }^{61}$

Although multidisciplinary management approaches to type 2 diabetes in adults improve glycemic control, ${ }^{62}$ evidence is lacking in children with type 2 diabetes. Multidisciplinary approaches to the management of type 2 diabetes in adolescents vary according to location, experience, local expertise, and availability of institutional and community resources. Individual components of a multidisciplinary team may include primary care physicians, endocrinologists, dieticians, certified diabetes educators, pharmacists, personal trainers or exercise physiologists, social workers, psychologists, and case managers or care coordinators. ${ }^{63}$ Although current clinical structure may provide selected services as part of routine care, it is the interaction among team members and the cohesive, focused management philosophy that adds true value to multidisciplinary care. ${ }^{52}$ However, the optimal components, organization, and interaction between team members are unknown.

\section{Adolescent and family members}

In patient-centered health care, adolescents with type 2 diabetes, parents, and family members are the center of the multidisciplinary health care team. The role of the patient, parent, and other family members in diabetes care varies with patient age and developmental stage. In young children, a family member will be the center of the health care team, whereas in adolescents and young adults, the patient will lead the team. As adolescents approach transition to adultoriented care, health care providers should partner with the family and other members of the health care team to facilitate progressive responsibility for self-management of diabetes 
with appropriate levels of parental oversight. This fosters independent self-care responsibility that is expressed through self-monitoring, utilization of blood glucose data, meal planning, activity goals, and problem solving. ${ }^{52,64}$ Creating and sustaining change are dependent on patient activation, and failure to acknowledge the need for change poses a substantial barrier to both patients and health care providers. ${ }^{52,64}$ Development of multidisciplinary diabetes education strategies designed to enhance self-efficacy and self-management may increase activation among adolescents and supporting family members and may enhance diabetes self-care including monitoring of blood glucose, analyzing glucose readings, adjusting dietary intake, modifying physical activity, and promoting weight loss. ${ }^{52,64}$ As self-efficacy increases, patients accept greater responsibility for their diabetes and the consequences of treatment choices. As this occurs, the multidisciplinary diabetes team focuses less on directing and dictating care and more on guiding and supporting individualized, collaborative patient management strategies. ${ }^{52,64}$

Adolescents with type 2 diabetes are unlikely to create and sustain changes in eating habits, physical activity, and lifestyle without the support of family members. Adolescents with type 2 diabetes come from families where obesity, inactive lifestyles, high-fat diets, binge eating, and parental diabetes are common, ${ }^{28}$ and poor control of type 2 diabetes in parents may be detrimental to diabetes control in adolescents. Having family members with diabetes has been identified by adolescents as both positive and negative influences on disease management depending on the disease management behaviors, disease complications observed, and expectations modeled by others. ${ }^{65}$ Parents with poorly controlled diabetes may be reluctant to encourage improved self-management in their children with diabetes and require additional assistance in facilitating diabetes self-management in their children. ${ }^{66}$ Adolescents with type 2 diabetes and their parents underestimate the degree of overweight or obesity, and this is associated with poorer exercise, dietary intake, and greater barriers to change. ${ }^{67}$ Additional barriers to change identified by adolescents with type 2 diabetes and their parents include a lack of perceived normalcy, especially with respect to food choices, environmental challenges at school and in the home, and the lack of opportunity to interact with other adolescents with type 2 diabetes ${ }^{65,66}$ Adolescents with type 2 diabetes generally have poor diet and exercise habits, even if medication monitoring and adherence are adequate. ${ }^{68}$ Effective, multidisciplinary disease management programs should account for family and social context, seek buy-in from the family unit, and engage both the patient and the family members responsible for care. Empowering change in family members has positive effects on diabetes management in adults. ${ }^{69}$ Collaborative participation in multidisciplinary disease management programs by the adolescent with diabetes and a parent with diabetes may create accountability and increase effectiveness if both adolescent and parent are activated and willing to change behavior. Family involvement in diabetes management strategies is associated with fewer unhealthy behaviors, ${ }^{70}$ and this may be instrumental for adolescents to make successful, sustainable lifestyle changes, as parents commonly purchase food, prepare meals, and model behavior for their children. ${ }^{69}$

\section{Primary care providers}

Primary care providers play an integral role in the multidisciplinary diabetes care team. By establishing long-term relationships with adolescents with diabetes and their families, primary care providers facilitate health promotion, health maintenance, disease prevention, counseling, patient education, and diagnosis and treatment of acute and chronic illnesses. ${ }^{71}$ Within the medical home and CCM, the primary care physician often organizes and coordinates care between members of the multidisciplinary care team. Primary care providers may be the only members of the health care team with training in the delivery of comprehensive, developmentally appropriate health care to adolescents and young adults. Although other members of the multidisciplinary team may have training to deliver developmentally appropriate services within their scope of practice, the primary care physician is capable of integrating and coordinating care across multiple services to ensure that a consistent, developmentally appropriate philosophy of care is provided to meet the needs of each patient and family. ${ }^{52,64}$

While many adult-oriented providers have experience and comfort in managing type 2 diabetes, providing diabetesrelated screenings, and monitoring for disease complications, pediatric-oriented providers may not. Primary care provider's knowledge of institutional, community, and regional diabetes management resources will facilitate assembly of the multidisciplinary care team. Coordination of services by primary care providers within a medical home model facilitates comprehensive diabetes care and may assist with transition and transfer of care from pediatric to adult health care systems. ${ }^{52,64}$

\section{Diabetes specialists}

Diabetes specialists, including endocrinologists and midlevel providers with advanced training in diabetes, can play important roles in the multidisciplinary management of type 2 diabetes; however, availability and accessibility in 
both industrialized and nonindustrialized countries may be limited. As the prevalence of type 2 diabetes in adolescents and young adults increases, ${ }^{12}$ the ability of diabetes specialists to manage type 2 diabetes in this population will be even further limited, and alternative approaches to diabetes care will be needed. Although specialist care may result in better diabetes-specific process measures, it has not been consistently demonstrated to improve survival in adults with type 2 diabetes. ${ }^{72,73}$ Children with type 2 diabetes may be more likely to receive care from diabetes specialists as a result of pediatric primary care providers limited comfort and experience caring for type 2 diabetes. However, it is currently unknown if care by diabetes specialists improves long-term outcomes in children with type 2 diabetes.

While diabetes specialists should be part of the multidisciplinary care team, they may not need to be involved in the management of routine type 2 diabetes if appropriate services and infrastructure are in place. Long-distance consultation via telephone, computer, or video conferencing with diabetes specialists may be sufficient when expert consultation is needed. Diabetes specialists may also enhance diabetes management within the team by designing and leading structured continuing medical education programs on medical management of type 2 diabetes, disease management guidelines, and prevention of secondary complications. Despite clinical practice guidelines set forth by the ADA and other expert panels, there remains wide variation in the management of type 2 diabetes among pediatric diabetes specialists. In a recent study, pediatric endocrinologists often failed to follow recommendations for screening and management of hypertension, hyperlipidemia, microalbuminuria, and foot care, and only $34 \%$ of providers were concordant with all guidelines. ${ }^{74}$ Although many reasons could explain this variation, there is significant room for improvement. Greater attention to type 2 diabetes guidelines in youth, enhanced provider education programs, and increased collaboration among primary care providers and diabetes specialists may enhance comprehensive diabetes management within the medical home and help accommodate the increasing burden of diabetes care on the health care system.

\section{Diabetes educators and nutritionists}

In order to create and sustain changes in lifestyle, diet, physical activity, and diabetes management, diabetes-related education and nutritional curricula are needed to establish a foundation of patient and provider knowledge upon which more comprehensive disease management strategies can build. Diabetes education is necessary but not sufficient to enhance self-management in patients with diabetes. ${ }^{75}$ Creation of diabetes education programs that are sensitive to each individual patient's level of literacy and numeracy may enhance the effectiveness of diabetes and nutrition education. Literacy- and numeracy-sensitive diabetes education programs ${ }^{76}$ have been shown to improve self-efficacy and diabetes control. ${ }^{77}$ The use of such tailored education programs may also improve safety and quality of care. ${ }^{78}$

Family-centered, group diabetes education programs, such as the Families, Adolescents, and Children's Teamwork Study (FACTS), ${ }^{79,80}$ have demonstrated significant improvement in glycemic control among adolescents type 1 diabetes. This clinic-integrated education program delivered by members of a multidisciplinary care team, including nurse specialists, physicians, and dieticians, addresses teamwork, communication, interdependence or shared responsibility for diabetes care, and letting go in addition to standard nutrition, physical activity, and glucose monitoring aspects of diabetes management. Such programs may provide a framework for the development of similar programs for adolescents with type 2 diabetes.

Diabetes education programs targeting adolescents with type 2 diabetes and their parents face unique challenges due to high rate of risk factors, lifestyle choices, and comorbid type 2 diabetes among family members. ${ }^{28}$ Families containing multiple members with type 2 diabetes demonstrate poor glycemic control among both parents and children, suggesting that interventions targeted at the adolescent alone may be insufficient. ${ }^{28} \mathrm{~A}$ standardized diabetes education program was developed for adolescents with type 2 diabetes and implemented at the time of enrollment for all participants in the TODAY study. ${ }^{75}$ Adolescents and a family member attend the sessions together. The curriculum is led by a certified diabetes educator and focuses on understanding disease physiology, disease management, and progressive skill building. Mastery of skills is measured by quizzes and required for progression through the program. Further studies on the effectiveness and implementation of such programs in adolescents with type 2 diabetes are needed.

\section{Behavioral counselors and activity coaches}

The availability of psychologists, counselors, therapists, and psychiatrists for consultation with the multidisciplinary diabetes management team is an important consideration for many patients. Adolescents with type 2 diabetes are often faced with the diagnosis of a chronic disease during a time of increasing autonomy. ${ }^{52,64}$ Stress, anxiety, depressed mood, 
and other emotional stressors may contribute to poor disease control in adolescents and young adults, and addressing these barriers may facilitate behavioral change and improve clinical outcomes. ${ }^{68}$

The TODAY study is currently utilizing Personal Activity and Nutrition Leaders (PALs) as a portion of the intensive lifestyle intervention program. Through regular contact with the adolescent and participating family support person, PALs encourage healthy eating, physical activity, and restructuring of the home environment. In addition to examining the effect of medication vs medication plus lifestyle modification, the study is collecting data on physical activity and accelerometer data to measure physical activity and fitness. ${ }^{81}$

The role of activity coaches and personal trainers within multidisciplinary diabetes care teams has not been well studied. Intensive lifestyle modification with diet and exercise is superior to treatment with metformin alone in preventing type 2 diabetes in adults with impaired glucose tolerance. ${ }^{82}$ Regular physical activity has been shown to reduce abdominal visceral fat, increase insulin sensitivity, decrease insulin resistance, improve lipid profiles, reduce inflammation, improve endothelial function, and reduce blood pressure in patients with type 2 diabetes. ${ }^{83}$ The 2008 US Department of Health and Human Services Physical Activity Guidelines recommended that children and adolescents should get at least 60 minutes of physical activity daily, with the majority of this time composed of moderate-vigorous intensity aerobic physical activity distributed over at least 3 days per week. ${ }^{83}$ However, in children, greater focus on reduction of sedentary activity and less focus on structured exercise programs appear to be more effective than organized high-intensity exercise. ${ }^{63}$

\section{Care coordinators, case managers, and social workers}

Utilization of case management and team management strategies in adults with type 2 diabetes results in superior glycemic control compared with isolated interventions targeting patient education and provider education or creating patient registries. ${ }^{62}$ Care coordinators enhance disease management by facilitating communication and coordination among providers, subspecialists, and other team members. Coordinated delivery of services among members of the health care team allows linkage of targeted patient and family diabetes education, demonstration of practical dietary and activity changes by nutrition and exercise specialists, reinforcement of clinical importance of disease control by providers, and follow-up by the care coordinator to address family-specific issues that may facilitate or pose barriers to change.
Linkage of the multidisciplinary team allows delivery of a unified philosophical diabetes management approach and provision of ongoing, real-time follow-up by care coordinators. Contact with the multidisciplinary team between scheduled clinical visits allows follow-up of home glucose monitoring data, dietary changes, activity goals, and medication titration to occur between scheduled clinic visits. Frequent interactions by telephone and computer-based technologies may increase patient accountability and adherence and may facilitate more rapid changes in the diabetes treatment plan to improve disease control. A recent study in adolescents with type 1 diabetes demonstrated improvement in self-management, problem solving, and glucose control through the use of an Internet portal. ${ }^{84}$ Utilization of such technology-based interfaces may be particularly useful in disease management of adolescents.

The role of care coordinator may be filled by nurses, case managers, pharmacists, social workers, or other individuals. Multidisciplinary diabetes management programs in adults utilizing care coordinators who are allowed to make medication changes independent of clinicians have greater reductions in $\mathrm{HbA}_{1 \mathrm{c}}$ levels $(0.96 \%$ vs $0.41 \%$ ) compared with care coordinators who were not independent. ${ }^{62}$ Utilizing clinical pharmacists as case managers or care coordinators within adult multidisciplinary diabetes teams creates significant reductions in $\mathrm{HbA}_{1}$ $(2.1 \%$ vs $0.9 \%) .{ }^{85}$ Thus, encouraging care coordinator independence with appropriate support from the multidisciplinary care team may be a useful strategy to enhance diabetes management and glucose control in adolescents with type 2 diabetes; however, studies demonstrating such findings in youth are lacking.

Utilization of community health workers as members of the multidisciplinary diabetes team has been shown to reduce emergency room visits and improve diabetes control in minority populations and underserved areas, suggesting that community-based, culturally tailored programs may enhance diabetes outcomes. ${ }^{86-88}$ Social workers are also important members of the multidisciplinary diabetes team and provide links to institutional, state, and community resources. Social workers can help identify local community centers, parks, and other recreational activities within the community to increase the physical activity of adolescents and families with type 2 diabetes. They may also be able to assist families with access issues and navigating insurance resources so that continuous insurance coverage can be arranged as adolescents age out of their parent's insurance policies. When adolescents do not have insurance, social workers can assist with enrollment in federally funded programs or help find indigent care clinics within the community. 


\section{Implementation of multidisciplinary management in type 2 diabetes}

Although the incidence and prevalence of type 2 diabetes in youth are increasing, ${ }^{2,3,20}$ the population density of adolescents with type 2 diabetes remains relatively low. This creates intrinsic difficulty in identifying patients, centralizing care within geographic areas, and establishing and sustaining clinic-based multidisciplinary diabetes care teams. Clinical follow-up among adolescents with type 2 diabetes is anecdotally low. ${ }^{89}$ Although the reasons for this are poorly understood, it may be related in part to the lack of acute events when the disease is poorly controlled and the relatively silent disease onset and progression early in the course of type 2 diabetes. Additionally, the implementation of multidisciplinary care teams, CCM principles, and PCMH infrastructure face payment and reimbursement challenges in start-up costs, daily operations, and long-term sustainability. At present, it is unclear if cost savings from such models will adequately cover operational costs to allow sustainability. ${ }^{49,90}$ Although these challenges may limit the development of clinic-based multidisciplinary care teams for adolescents with diabetes, multidisciplinary management principles are applicable and may increase the quality of care delivered. Utilization of technology-based communication, education strategies, and disease management tools via telephone, computer, Internet, ${ }^{84}$ or texting may provide novel approaches to diabetes management without dependence on traditional face-to-face clinical encounters.

In spite of evidence supporting the use of multidisciplinary management strategies in adults with type 2 diabetes ${ }^{62}$ published program descriptions, implementation studies, and program evaluations are lacking in adolescents with type 2 diabetes. Furthermore, information on the safety, efficacy, and long-term consequences of type 2 diabetes treatment and disease progression in youth is lacking. To date, the TODAY study is the largest study of type 2 diabetes in youth, and it should provide critical information on the natural history of disease in youth and the comparative effectiveness of medication and lifestyle interventions in youth with type 2 diabetes. ${ }^{81}$ This study, although rigorously controlled and defined, contains many aspects of a multidisciplinary care team, including participation by adolescents and a family support person in diabetes management; utilization of diabetes educators with curricula geared towards both adolescents and caregivers; intensive lifestyle intervention with personal activity, behavioral, and nutrition coaches; and management of comorbid conditions, such as dyslipidemia, hypertension, and microalbuminuria. ${ }^{81}$ Lessons learned from this study could significantly inform the development of disease management programs in adolescents with type 2 diabetes.

\section{Future work}

If experience with multidisciplinary management of type 2 diabetes in adults holds true, creation of multidisciplinary care teams for type 2 diabetes in adolescents may improve disease control and delay the development of diabetes-related complications. Given the relatively low, although increasing, prevalence of type 2 diabetes in youth, multidisciplinary services will likely need to coordinate and organize care over broad geographic areas. Although the creation of in-clinic and on-site multidisciplinary teams may not be practical or economical in all areas, development of hybrid, multidisciplinary diabetes management strategies combining on-site clinical encounters with off-site remote disease management may facilitate more comprehensive, organized, convenient, and economical care. ${ }^{90}$ Utilization of electronic medical records and development of technology interfaces utilizing cellular phones, computers, Internet resources, and social networking may facilitate engagement of adolescents in disease management and improve diabetes control. Development, implementation, and evaluation of multidisciplinary care models in youth with type 2 diabetes are urgently needed, and experiences from disease management programs for adults with type 2 diabetes should facilitate development.

\section{Disclosure}

The authors report no conflicts of interest in this work.

\section{References}

1. American Diabetes Association. Type 2 diabetes in children and adolescents. Diabetes Care. 2000;23(3):381-389.

2. Alberti G, Zimmet P, Shaw J, Bloomgarden Z, Kaufman F, Silink M. Type 2 diabetes in the young: the evolving epidemic - the international diabetes federation consensus workshop. Diabetes Care. 2004;27(7):1798-1811.

3. Rosenbloom AL, Joe JR, Young RS, Winter WE. Emerging epidemic of type 2 diabetes in youth. Diabetes Care. 1999;22(2):345-354.

4. Zeitler P. Update on nonautoimmune diabetes in children. J Clin Endocrinol Metab. 2009;94(7):2215-2220.

5. American Diabetes Association. Diagnosis and classification of diabetes mellitus. Diabetes Care. 2008;31 Suppl 1:S55-S60.

6. Hathout EH, Thomas W, El-Shahawy M, Nahab F, Mace JW. Diabetic autoimmune markers in children and adolescents with type 2 diabetes. Pediatrics. 2001;107(6):E102.

7. Fagot-Campagna A, Pettitt DJ, Engelgau MM, et al. Type 2 diabetes among North American children and adolescents: an epidemiologic review and a public health perspective. J Pediatr. 2000;136(5):664-672.

8. American Diabetes Association. Type 2 diabetes in children and adolescents. Pediatrics. 2000;105(3 Pt 1):671-680.

9. Wang J, Miao D, Babu S, et al. Prevalence of autoantibody-negative diabetes is not rare at all ages and increases with older age and obesity. J Clin Endocrinol Metab. 2007;92(1):88-92. 
10. Reinehr T, Schober E, Wiegand S, Thon A, Holl R. Beta-cell autoantibodies in children with type 2 diabetes mellitus: subgroup or misclassification? Arch Dis Child. 2006;91(6):473-477.

11. Tfayli H, Bacha F, Gungor N, Arslanian S. Phenotypic type 2 diabetes in obese youth: insulin sensitivity and secretion in islet cell antibodynegative versus -positive patients. Diabetes. 2009;58(3):738-744.

12. Pinhas-Hamiel O, Zeitler P. The global spread of type 2 diabetes mellitus in children and adolescents. J Pediatr. 2005;146(5):693-700.

13. Dabelea D, Hanson RL, Bennett PH, Roumain J, Knowler WC, Pettitt DJ. Increasing prevalence of Type II diabetes in American Indian children. Diabetologia. 1998;41(8):904-910.

14. Liese AD, D'Agostino RB Jr, Hamman RF, et al. The burden of diabetes mellitus among US youth: prevalence estimates from the SEARCH for Diabetes in Youth Study. Pediatrics. 2006;118(4):1510-1518.

15. Dabelea D, Bell RA, D'Agostino RB Jr, et al. Incidence of diabetes in youth in the United States. JAMA. 2007;297(24):2716-2724.

16. Urakami T, Morimoto S, Nitadori Y, Harada K, Owada M, Kitagawa T. Urine glucose screening program at schools in Japan to detect children with diabetes and its outcome-incidence and clinical characteristics of childhood type 2 diabetes in Japan. Pediatr Res. 2007;61(2):141-145.

17. Wei JN, Sung FC, Lin CC, Lin RS, Chiang CC, Chuang LM. National surveillance for type 2 diabetes mellitus in Taiwanese children. JAMA. 2003;290(10):1345-1350.

18. Craig ME, Femia G, Broyda V, Lloyd M, Howard NJ. Type 2 diabetes in indigenous and non-indigenous children and adolescents in New South Wales. Med J Aust. 2007;186(10):497-499.

19. Ehtisham S, Kirk J, McEvilly A, et al. Prevalence of type 2 diabetes in children in Birmingham. BMJ. 2001;322(7299):1428.

20. Goran MI, Davis J, Kelly L, et al. Low prevalence of pediatric type 2 diabetes: where's the epidemic? J Pediatr. 2008;152(6):753-755.

21. Matyka KA. Type 2 diabetes in childhood: epidemiological and clinical aspects. Br Med Bull. 2008;86:59-75.

22. Bobo N, Evert A, Gallivan J, et al. An update on type 2 diabetes in youth from the National Diabetes Education Program. Pediatrics. 2004;114(1):259-263.

23. Hannon TS, Rao G, Arslanian SA. Childhood obesity and type 2 diabetes mellitus. Pediatrics. 2005;116(2):473-480.

24. Rodbard HW. Diabetes screening, diagnosis, and therapy in pediatric patients with type 2 diabetes. Medscape J Med. 2008;10(8):184; quiz 184

25. Kaufman FR. Type 2 diabetes in children and youth. Endocrinol Metab Clin North Am. 2005;34(3):659-676, ix-x.

26. Dean HJ, Young TK, Flett B, Wood-Steiman P. Screening for type-2 diabetes in aboriginal children in northern Canada. Lancet. 1998; 352(9139):1523-1524.

27. Jiang X, Srinivasan SR, Radhakrishnamurthy B, Dalferes ER, Berenson GS. Racial (black-white) differences in insulin secretion and clearance in adolescents: the Bogalusa heart study. Pediatrics. 1996;97(3):357-360.

28. Pinhas-Hamiel O, StandifordD, Hamiel D, Dolan LM, Cohen R, Zeitler PS. The type 2 family: a setting for development and treatment of adolescent type 2 diabetes mellitus. Arch Pediatr Adolesc Med. 1999; 153(10):1063-1067.

29. Kitagawa T, Owada M, Urakami T, Yamauchi K. Increased incidence of non-insulin dependent diabetes mellitus among Japanese schoolchildren correlates with an increased intake of animal protein and fat. Clin Pediatr (Phila). 1998;37(2):111-115.

30. Schulze MB, Manson JE, Ludwig DS, et al. Sugar-sweetened beverages, weight gain, and incidence of type 2 diabetes in young and middle-aged women. JAMA. 2004;292(8):927-934.

31. Malik VS, Schulze MB, Hu FB. Intake of sugar-sweetened beverages and weight gain: a systematic review. Am J Clin Nutr. 2006;84(2): 274-288.

32. Pettitt DJ, Nelson RG, Saad MF, Bennett PH, Knowler WC. Diabetes and obesity in the offspring of Pima Indian women with diabetes during pregnancy. Diabetes Care. 1993;16(1):310-314.
33. Phillips DI. Birth weight and the future development of diabetes. A review of the evidence. Diabetes Care. 1998;21 Suppl 2:B15-B155.

34. International Expert Committee. International Expert Committee report on the role of the A1C assay in the diagnosis of diabetes. Diabetes Care. 2009;32(7):1327-1334.

35. American Diabetes Association. Screening for type 2 diabetes. Diabetes Care. 2004;27 Suppl 1:S11-S14.

36. Williams DE, Cadwell BL, Cheng YJ, et al. Prevalence of impaired fasting glucose and its relationship with cardiovascular disease risk factors in US adolescents, 1999-2000. Pediatrics. 2005;116(5): 1122-1126.

37. Anand SG, Mehta SD, Adams WG. Diabetes mellitus screening in pediatric primary care. Pediatrics. 2006;118(5):1888-1895.

38. Hillier TA, Pedula KL. Complications in young adults with early-onset type 2 diabetes: losing the relative protection of youth. Diabetes Care. 2003;26(11):2999-3005.

39. Pinhas-Hamiel O, Zeitler P. Acute and chronic complications of type 2 diabetes mellitus in children and adolescents. Lancet. 2007; 369(9575):1823-1831.

40. Fagot-Campagna A, Knowler WC, Pettitt DJ. Type 2 diabetes in Pima Indian children: cardiovascular risk factors at diagnosis and 10 years later. Diabetes. 1998;47:A155-A155.

41. Eppens MC, Craig ME, Cusumano J, et al. Prevalence of diabetes complications in adolescents with type 2 compared with type 1 diabetes. Diabetes Care. 2006;29(6):1300-1306.

42. Yokoyama H, Okudaira M, Otani T, et al. Existence of early-onset NIDDM Japanese demonstrating severe diabetic complications. Diabetes Care. 1997;20(5):844-847.

43. Karabouta Z, Barnett S, Shield JP, Ryan FJ, Crowne EC. Peripheral neuropathy is an early complication of type 2 diabetes in adolescence. Pediatr Diabetes. 2008;9(2):110-114.

44. Kawahara R, Amemiya T, Yoshino M, Miyamae M, Sasamoto K, OmoriY. Dropout of young non-insulin-dependent diabetics from diabetic care. Diabetes Res Clin Pract. 1994;24(3):181-185.

45. Jones KL, Arslanian S, Peterokova VA, Park JS, Tomlinson MJ. Effect of metformin in pediatric patients with type 2 diabetes: a randomized controlled trial. Diabetes Care. 2002;25(1):89-94.

46. Gottschalk M, Danne T, Vlajnic A, Cara JF. Glimepiride versus metformin as monotherapy in pediatric patients with type 2 diabetes: a randomized, single-blind comparative study. Diabetes Care. 2007; 30(4):790-794.

47. Dean HJ. Dancing with many different ghosts: treatment of youth with type 2 diabetes. Diabetes Care. 2002;25(1):237-238.

48. Homer CJ, Klatka K, Romm D, et al. A review of the evidence for the medical home for children with special health care needs. Pediatrics. 2008;122(4):e922-e937.

49. Carrier E, Gourevitch MN, Shah NR. Medical homes: challenges in translating theory into practice. Med Care. 2009;47(7):714-722.

50. Berenson RA, Hammons T, Gans DN, et al. A house is not a home: keeping patients at the center of practice redesign. Health Aff (Millwood). 2008;27(5):1219-1230.

51. Wagner EH, Austin BT, Davis C, Hindmarsh M, Schaefer J, Bonomi A. Improving chronic illness care: translating evidence into action. Health Aff (Millwood). 2001;20(6):64-78.

52. Brink SJ, Chiarelli FG. Education and multidisciplinary team approach in childhood diabetes. Acta Biomed. 2004;75(1):7-21.

53. Berwick DM, Nolan TW, Whittington J. The triple aim: care, health, and cost. Health Aff (Millwood). 2008;27(3):759-769.

54. Forrest CB, Simpson L, Clancy C. Child health services research. Challenges and opportunities. JAMA. 1997;277(22):1787-1793.

55. Ofman JJ, Badamgarav E, Henning JM, et al. Does disease management improve clinical and economic outcomes in patients with chronic diseases? A systematic review. Am J Med. 2004;117(3):182-192.

56. Renders CM, Valk GD, Griffin SJ, Wagner EH, Eijk Van JT, Assendelft WJ. Interventions to improve the management of diabetes in primary care, outpatient, and community settings: a systematic review. Diabetes Care. 2001;24(10):1821-1833. 
57. Sadur CN, Moline N, Costa M, et al. Diabetes management in a health maintenance organization. Efficacy of care management using cluster visits. Diabetes Care. 1999;22(12):2011-2017.

58. Renders CM, Valk GD, Griffin S, Wagner EH, Eijk JT, Assendelft WJ. Interventions to improve the management of diabetes mellitus in primary care, outpatient and community settings. Cochrane Database Syst Rev. 2001;(1):CD001481.

59. Rothman RL, Malone R, Bryant B, et al. A randomized trial of a primary care-based disease management program to improve cardiovascular risk factors and glycated hemoglobin levels in patients with diabetes. Am J Med. 2005;118(3):276-284.

60. Rothman RL, So SA, Shin J, et al. Labor characteristics and program costs of a successful diabetes disease management program. Am J Manag Care. 2006;12(5):277-283.

61. Wagner EH, Sandhu N, Newton KM, McCulloch DK, Ramsey SD, Grothaus LC. Effect of improved glycemic control on health care costs and utilization. JAMA. 2001;285(2):182-189.

62. Shojania KG, Ranji SR, McDonald KM, et al. Effects of quality improvement strategies for type 2 diabetes on glycemic control: a meta-regression analysis. JAMA. 2006;296(4):427-440.

63. Berry D, Urban A, Grey M. Management of type 2 diabetes in youth (part 2). J Pediatr Health Care. 2006;20(2):88-97.

64. Brink SJ, Miller M, Moltz KC. Education and multidisciplinary team care concepts for pediatric and adolescent diabetes mellitus. J Pediatr Endocrinol Metab. 2002;15(8):1113-1130.

65. Mulvaney SA, Mudasiru E, Schlundt DG, et al. Self-management in type 2 diabetes: the adolescent perspective. Diabetes Educ. 2008;34(4):674-682.

66. Mulvaney SA, Schlundt DG, Mudasiru E, et al. Parent perceptions of caring for adolescents with type 2 diabetes. Diabetes Care. 2006; 29(5):993-997.

67. Skinner AC, Weinberger M, Mulvaney S, Schlundt D, Rothman RL. Accuracy of perceptions of overweight and relation to self-care behaviors among adolescents with type 2 diabetes and their parents. Diabetes Care. 2008;31(2):227-229.

68. Rothman RL, Mulvaney S, Elasy TA, et al. Self-management behaviors, racial disparities, and glycemic control among adolescents with type 2 diabetes. Pediatrics. 2008;121(4):e912-e919.

69. Cole I, Chesla CA. Interventions for the family with diabetes. Nurs Clin North Am. 2006;41(4):625-639, vii.

70. Bradshaw B. The role of the family in managing therapy in minority children with type 2 diabetes mellitus. J Pediatr Endocrinol Metab. 2002;15 Suppl 1:547-551.

71. AAFP.org [homepage on the Internet]. American Academy of Family Physicians, Inc; c2010. Available from: http://www.aafp.org/ online/en/home/policy/policies/p/primarycare.html. Accessed May 9, 2010 .

72. McAlister FA, Majumdar SR, Eurich DT, Johnson JA. The effect of specialist care within the first year on subsequent outcomes in 24,232 adults with new-onset diabetes mellitus: population-based cohort study. Qual Saf Health Care. 2007;16(1):6-11.

73. de Berardis G, Pellegrini F, Franciosi M, et al. Quality of care and outcomes in type 2 diabetic patients: a comparison between general practice and diabetes clinics. Diabetes Care. 2004;27(2):398-406.

74. Wong K, Potter A, Mulvaney S, Russell WE, Schlundt DG, Rothman RL. Pediatric endocrinologists' management of children with type 2 diabetes. Diabetes Care. 2010;33(3):512-514.

Journal of Multidisciplinary Healthcare

\section{Publish your work in this journal}

The Journal of Multidisciplinary Healthcare is an international, peerreviewed open-access journal that aims to represent and publish research in healthcare areas delivered by practitioners of different disciplines. This includes studies and reviews conducted by multidisciplinary teams as well as research which evaluates the results or conduct of such teams or Submit your manuscript here: http://www.dovepress.com/journal-of-multidisciplinary-healthcare-journal
75. Grey M, Schreiner B, Pyle L. Development of a diabetes education program for youth with type 2 diabetes. Diabetes Educ. 2009; 35(1):108-116.

76. Wolff K, Cavanaugh K, Malone R, et al. The Diabetes Literacy and Numeracy Education Toolkit (DLNET): materials to facilitate diabetes education and management in patients with low literacy and numeracy skills. Diabetes Educ. 2009;35(2):233-236, 238-241, 244-245.

77. Cavanaugh K, Wallston KA, Gebretsadik T, et al. Addressing literacy and numeracy to improve diabetes care: two randomized controlled trials. Diabetes Care. 2009;32(12):2149-2155.

78. Rothman RL, Yin HS, Mulvaney S, Co JP, Homer C, Lannon C. Health literacy and quality: focus on chronic illness care and patient safety. Pediatrics. 2009;124 Suppl 3:S315-S326.

79. Murphy HR, Wadham C, Rayman G, Skinner TC. Approaches to integrating paediatric diabetes care and structured education: experiences from the Families, Adolescents, and Children's Teamwork Study (FACTS). Diabet Med. 2007;24(11):1261-1268.

80. Murphy HR, Wadham C, Rayman G, Skinner CT. Integrating pediatric diabetes education into routine clinical care: the Families, Adolescents and Children's Teamwork Study (FACTS). Diabetes Care. 2006; 29(5):1177.

81. Zeitler P, Epstein L, Grey M, et al. Treatment options for type 2 diabetes in adolescents and youth: a study of the comparative efficacy of metformin alone or in combination with rosiglitazone or lifestyle intervention in adolescents with type 2 diabetes. Pediatr Diabetes. 2007; 8(2):74-87.

82. Knowler WC, Barrett-Connor E, Fowler SE, et al. Reduction in the incidence of type 2 diabetes with lifestyle intervention or metformin. N Engl J Med. 2002;346(6):393-403.

83. Weltman NY, Saliba SA, Barrett EJ, Weltman A. The use of exercise in the management of type 1 and type 2 diabetes. Clin Sports Med. 2009; 28(3):423-439.

84. Mulvaney SA, Rothman RL, Wallston KA, Lybarger C, Dietrich MS. An internet-based program to improve self-management in adolescents with type 1 diabetes. Diabetes Care. 2010;33(3):602-604.

85. Choe HM, Mitrovich S, Dubay D, Hayward RA, Krein SL, Vijan S. Proactive case management of high-risk patients with type 2 diabetes mellitus by a clinical pharmacist: a randomized controlled trial. Am J Manag Care. 2005;11(4):253-260.

86. Gary TL, Batts-Turner M, Yeh HC, et al. The effects of a nurse case manager and a community health worker team on diabetic control, emergency department visits, and hospitalizations among urban African Americans with type 2 diabetes mellitus: a randomized controlled trial. Arch Intern Med. 2009;169(19):1788-1794.

87. Gary TL, Bone LR, Hill MN, et al. Randomized controlled trial of the effects of nurse case manager and community health worker interventions on risk factors for diabetes-related complications in urban African Americans. Prev Med. 2003;37(1):23-32.

88. Kim MT, Han HR, Song HJ, et al. A community-based, culturally tailored behavioral intervention for Korean Americans with type 2 diabetes. Diabetes Educ. 2009;35(6):986-994.

89. Reinehr T, Schober E, Roth CL, Wiegand S, Holl R. Type 2 diabetes in children and adolescents in a 2-year follow-up: insufficient adherence to diabetes centers. Horm Res. 2008;69(2):107-113.

90. Cavanaugh KL, White RO, Rothman RL. Exploring disease management programs for diabetes mellitus. Disease Management and Health Outcomes. 2007;15(2):73-81.

healthcare processes in general. The journal covers a wide range of areas and welcomes submission from practitioners at all levels, from all over the world. The manuscript management system is completely online and includes a very quick and fair peer-review system. Visit http://www.dovepress.com/testimonials.php to read real quotes from published authors. 